\title{
$1 \quad$ Flashing LEDs for microalgal production
}

2 Peter S.C. Schulze ${ }^{1,2}$, Rui Guerra ${ }^{3}$, Hugo Pereira ${ }^{2}$, Lisa M. Schüler ${ }^{2}$ and João C.S. Varela ${ }^{2 *}$ 3

$4 \quad{ }^{1}$ Faculty of Biosciences and Aquaculture, Nord University, 8049 Bodø, Norway

$5{ }^{2}$ Centre of Marine Sciences, University of Algarve, Campus de Gambelas, 8005-139 Faro,

6 Portugal

$7 \quad{ }^{3}$ Centro de Electrónica, Optoelectrónica e Telecomunicações, University of Algarve, Campus 8 de Gambelas, 8005-139 Faro, Portugal.

9

$10 *$ Corresponding author. Tel.: +351-289800051. Email address: jvarela@ualg.pt (J. Varela).

11 Key words: Linear electron transfer chain; pulsed artificial light; light attenuation, 12 photosynthesis; microalgae; non-photochemical quenching. 


\section{Abstract}

14 Flashing lights are next-generation tools to mitigate light attenuation and increase the 15 photosynthetic efficiency of microalgal cultivation systems illuminated by light emitting diodes 16 (LEDs). Optimal flashing light conditions depend on the reaction kinetics and properties of the 17 linear electron transfer chain, energy dissipation and storage mechanisms of a phototroph. In 18 particular, extreme short and intense light flashes potentially mitigate light attenuation in 19 photobioreactors without impairing photosynthesis. Intelligently controlling flashing light units 20 and selecting electronic components can maximise light emission and energy efficiency. We 21 discuss the biological, physical and technical properties of flashing luminaries for algal 22 production. We combine recent findings about photosynthetic pathways, self-shading in 23 photobioreactors and developments in solid-state technology towards the biotechnological 24 application of LEDs to microalgal production. 


\section{Artificial light in microalgal production}

Microalgae are a promising biological resource for the mass production of lipids, sugars, polymers or proteins for the food, feed and chemical industries [1]. The co-production of highvalue biomolecules such as polyunsaturated fatty acids, carotenoids, beta-glucans and phycobiliproteins for nutraceutical, pharmaceutical and biomedical applications increases the value of microalgal biomass and the economic feasibility of microalgae-based biorefineries [2]. Presently, high production costs of $5-25 € \mathrm{Kg}^{-1}$ hinders the economic feasibility of microalgalbased commodities [3, 4]. The European Commission supports research and development of microalgal biotechnology by allocating between 2007 and 2017 ca. 40 million $€$ annually to microalgae-based ventures. For photo- and mixotrophic (see Glossary Box for definitions) microalgal cultivation, light is one of the most important growth parameters; it can come from natural (sun) or artificial (lamps) sources [4, 5]. Although artificial light costs more than sunlight, it allows tight control of microalgal biochemistry and growth, increasing the reliability of industrial processes for the production of high value biomolecules [4, 5].

However, the competitiveness of any artificial light-driven microalgal production hinges on energy consumption. A decrease of energy costs requires improvements in photon harvesting by microalgae and the photon conversion efficiency of light sources. Better light energy usage by phototrophs can be achieved by tailoring species-specific emission spectra of artificial light sources $[5,6]$. Another strategy concerns not the light quality, but rather light delivery. Instead of using continuous illumination, recent studies propose using flashing lights (Fig. 1). Flashing light is intermittent light that can provide highly intense light flashes with a short duration (hereafter called light flash period or $t_{l}$ ) alternating with extended dark periods $\left(t_{d}\right)$. One flash period followed by a dark period can be defined as a flashing cycle $\left(t_{c}\right.$, in which $\left.t_{c}=t_{l}+t_{d}\right)$. The use of high light flash intensities $\left(I_{l}\right)$ enables light to penetrate deeper into the culture and mitigate light attenuation [7-9] in photobioreactors, maintaining a high photosynthetic efficiency of concentrated cultures $[10,11]$. To prevent photo-damage and inhibition of the phototroph under cultivation by too intense light flashes, the repetition rate of the light-dark transition (i.e., flashing light frequency, $f$ ) and the relative proportion of the light flash period (i.e., the duty cycle, $\varnothing$ ) within the flashing cycle should be adjusted to the biological reaction kinetics of photosynthetic processes and energy dissipation mechanisms (also often referred to as non-photochemical quenching or simply NPQ). Nonetheless, well-engineered luminaries are essential to emit efficiently flashing light regimes that are advantageous for phototrophic cultivation [4, 12]. Balancing these factors, flashing light can result into higher growth performance per input energy as if the same light energy is supplied in a continuous way [4, 

of (i) biological, (ii) physical and (iii) technical factors that are crucial for applying this promising tool to microalgal cultivation.

\section{Flashing light}

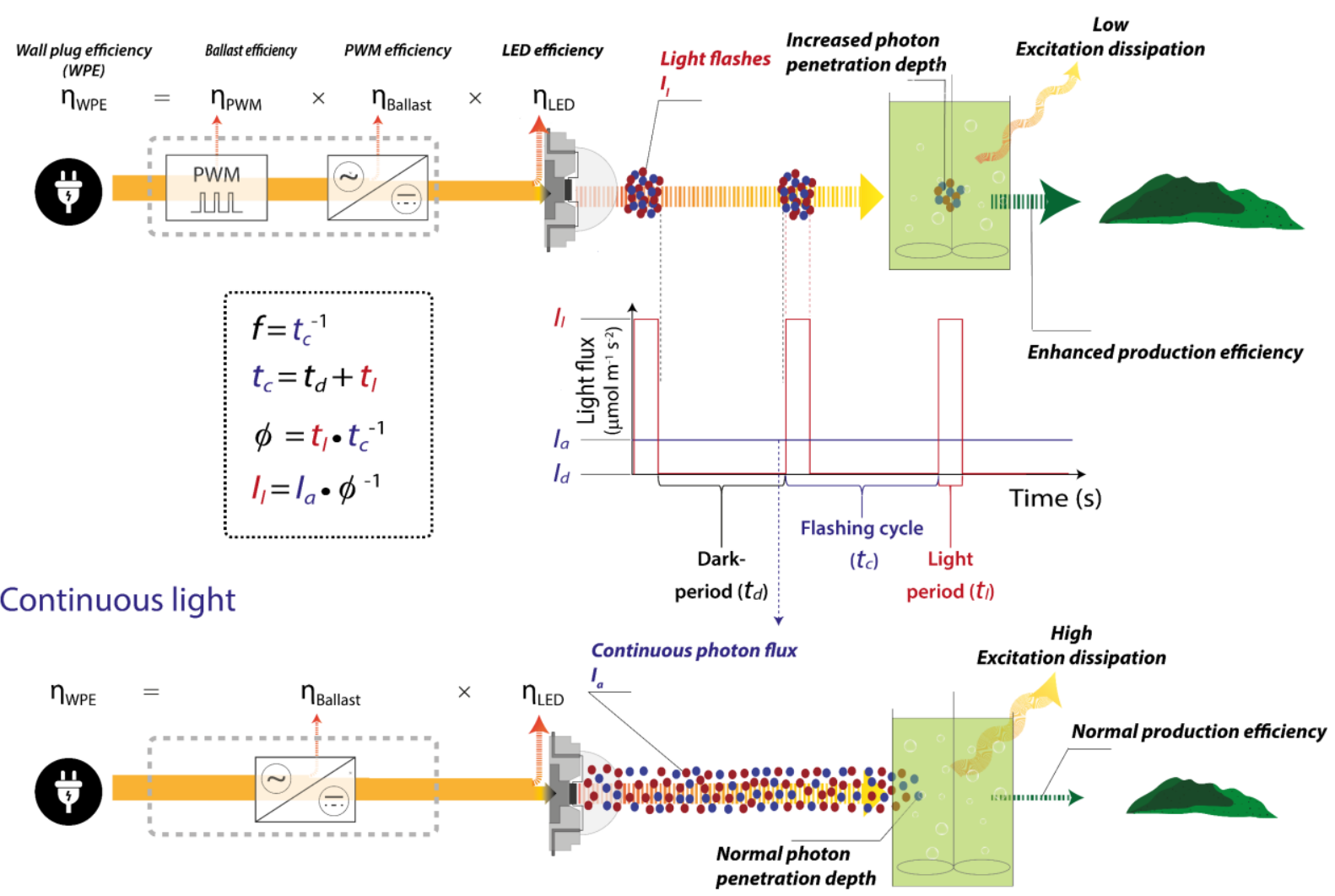

Figure 1 - Simplified diagram of a microalgal production unit using flashing (upper panel) and continuous light (bottom panel) emitted by light emitting diodes (LEDs). Flashing or pulsed light can increase light penetration depth and decrease excitation dissipation mechanisms, improving biomass productivity. A flashing light emitting system can transmit tailored wavebands that increase further photon penetration depth (e.g. green light for chlorophytes and Stramenopiles-Alveolata-Rhizaria species) or stimulate metabolic pathways and biochemical composition (e.g. blue and red light). The wall-plug efficiency (nwPE) is the product of the efficiency factors of all devices between power source and light output, including efficiencies of ballast ( $\eta_{\text {Ballast }}$ ), pulse-width modulator ( $\eta_{\text {PWM }}$ ) and LED ( $\left.\eta_{\text {LED }}\right)$. Flashing light devices emit light flash $\left(t_{l}\right)$ and dark periods $\left(t_{d}\right)$ in an approximately rectangular waveform. This waveform is commonly described by the flashing light frequency $(f)$ and duty cycle $(\phi)$. The frequency (in Hertz, $\mathrm{Hz}$ ) is the number of light-dark intervals (flashing cycles, $t_{c}$ ) that occur per second $\left(\mathrm{s}^{-1}\right)$. The duty cycle is the ratio between the flash period and the whole flashing cycle. The light intensity (in $\mu$ mol photons $\mathrm{m}^{-2} \mathrm{~s}^{-1}$ ) during $t_{l}$ is defined as flash intensity $\left(I_{l}\right)$, while during $t_{d}$ no 
78 light is emitted (e.g., $I_{d}=0 \mu \mathrm{mol}$ photons $\mathrm{m}^{-2} \mathrm{~s}^{-1}$ ). Under this condition, the time-averaged light 79 intensity $\left(I_{a}\right)$ during one flashing cycle can be expressed as $I_{a}=\emptyset \times I_{l}$ and is used to compare 80 flashing with continuous light treatments. Moreover, the flash intensity and the duty cycle are 81 inversely proportional at a given averaged light intensity. 


\section{Biological boundaries}

Microalgal cultures can display similar or higher photosynthetic rates under flashing light than under continuous light at the same averaged light intensity. This is referred to as "flashing light effect" [13, 14] or "light integration effect" [15] and occurs if the photosynthetic apparatus is working close to its full capacity (biological factor). However, in cultures with light attenuation, the flashing light effect can additionally be achieved by enhanced light delivery into the culture (physical factor), even though the photosynthetic apparatus is working at rates that are far lower than its full capacity. In this section, we define the flashing light conditions (e.g. frequency or duty cycle) at which the photosynthetic apparatus perceives a flashing light effect as a biological boundary. Generally, the flashing light effect refers to the response of a phototroph to timeaveraged light intensity $\left(I_{a}\right)$ during the flashing cycle, and not to the instantaneous light intensity of the light $\left(I_{l}\right)$ or dark $\left(I_{d}\right)$ periods (Fig. 1). When exposed to too low frequencies (e.g. $f=1$ $10 \mathrm{~Hz}$ ) with too low duty cycles (e.g., $\varnothing>0.5$ ), no flashing light effect takes place and phototrophs quench excess energy during the light period, and might experience enhanced respiration during the dark period (e.g., post-illumination respiration; $[13,15,16])$. This results in less growth and biomass losses. Moreover, molecular responses to stress in the phototroph under cultivation can also be activated.

The biological boundary depends on reaction kinetics of energy dissipation mechanisms, energy storage and the linear electron transfer chain (see Box 1). Flashing light studies on single leaves of land plants or microalgal cultures with low light attenuation potential can identify the biological boundary, and may be described as a function of frequency $(f)$ and duty cycle $(\varnothing)$, i.e. $f_{(\varnothing, \mathrm{f})}[17,18]$. For example, Jishi et al. [17] identified such function of the flashing light effect for lettuce (Lactuca sativa). Interestingly, their model also fits the photosynthetic performances of microalgal cultures with low biomass concentrations $\left(<0.1 \mathrm{~g} \mathrm{~L}^{-1}\right)$ or short light path lengths $(<1-2 \mathrm{~cm})$ and for land plants (e.g. tomato) under various flashing light conditions [19-27].

\subsection{Excitation dissipation and energy storage efficiencies}

Absorbed light energy is able to bring chlorophyll from its ground state $(\mathrm{Chl})$ to a singlet excited state $\left({ }^{1} \mathrm{Chl} *\right) .{ }^{1} \mathrm{Chl} *$ can pass its excitation energy via resonance or excitation energy transfer to adjacent chlorophyll molecules in the light harvesting complexes or the reaction centres of photosystem I or II (PSI or PSII). In the reaction centres, charge separation takes place and excitons can be photochemically quenched by provoking the transfer of electrons to the photosynthetic linear or cyclic electron transfer chains [28]. These photosynthetic pathways 
116 are essential for the production of adenosine triphosphate (ATP) and reducing equivalents such 117 as plastoquinol and nicotinamide adenine dinucleotide phosphate (NADPH; [28]). If the 118 reaction centres are "closed", i.e. if they are not able to process photon excess under high light 119 conditions, ${ }^{1} \mathrm{Chl} *$ can dissipate absorbed energy as heat through excitation dissipation 120 mechanisms or re-emit a photon (fluorescence) when falling back to its ground state (Chl). Both 121 processes prevent the formation of triplet $\mathrm{Chl}\left({ }^{3} \mathrm{Chl} *\right)$, which causes reactive oxygen species 122 (ROS) evolution [28]. If, for example, the storage capacity for reducing equivalents cannot cope 123 with the excess electrons under high light, the likelihood of ROS accumulation increases. Such 124 high ROS levels suppress protein synthesis, which is essential for repairing PSII upon photodamage [29]. To avoid excess ROS evolution during high light (flash) periods and maintain their metabolism during prolonged dark periods, phototrophs employ different energy

127 quenching and storage strategies under flashing light with a low duty cycle (e.g., $\varnothing<0.1$; Fig. 2;

$128[14,16,30,31])$. Usually, alternation between light and dark periods longer than seconds, 129 minutes or hours (implying frequencies $<1 \mathrm{~Hz}$ ) are referred to light that is supplied 130 intermittently, discontinuously or through light/dark cycles or photoperiods. For the sake of 131 convenience, however, the term "flashing light" and associated parameters will be used in all 132 time scales.

133 In this context, mechanisms of short-term energy storage (fs-ps time scale) follow the laws 134 of quantum dynamics and energy transport takes place via quantum coherence. The excitation 135 energy delivered by fs-ps lasting high light flashes can be stored in pigment cofactors (e.g. 136 chlorophylls, carotenoids or phylloquinones) as excitons or through inter-protein hopping 137 within the light harvesting complexes [32]. If reaction time permits, energy may be stored in 138 reaction centre II. In this time scale, excess energy may be dissipated through ultrafast reacting 139 quenchers (e.g. chlorophyll $a$; [32, 33]), resulting in pigment internal thermal decay or 140 fluorescence. 


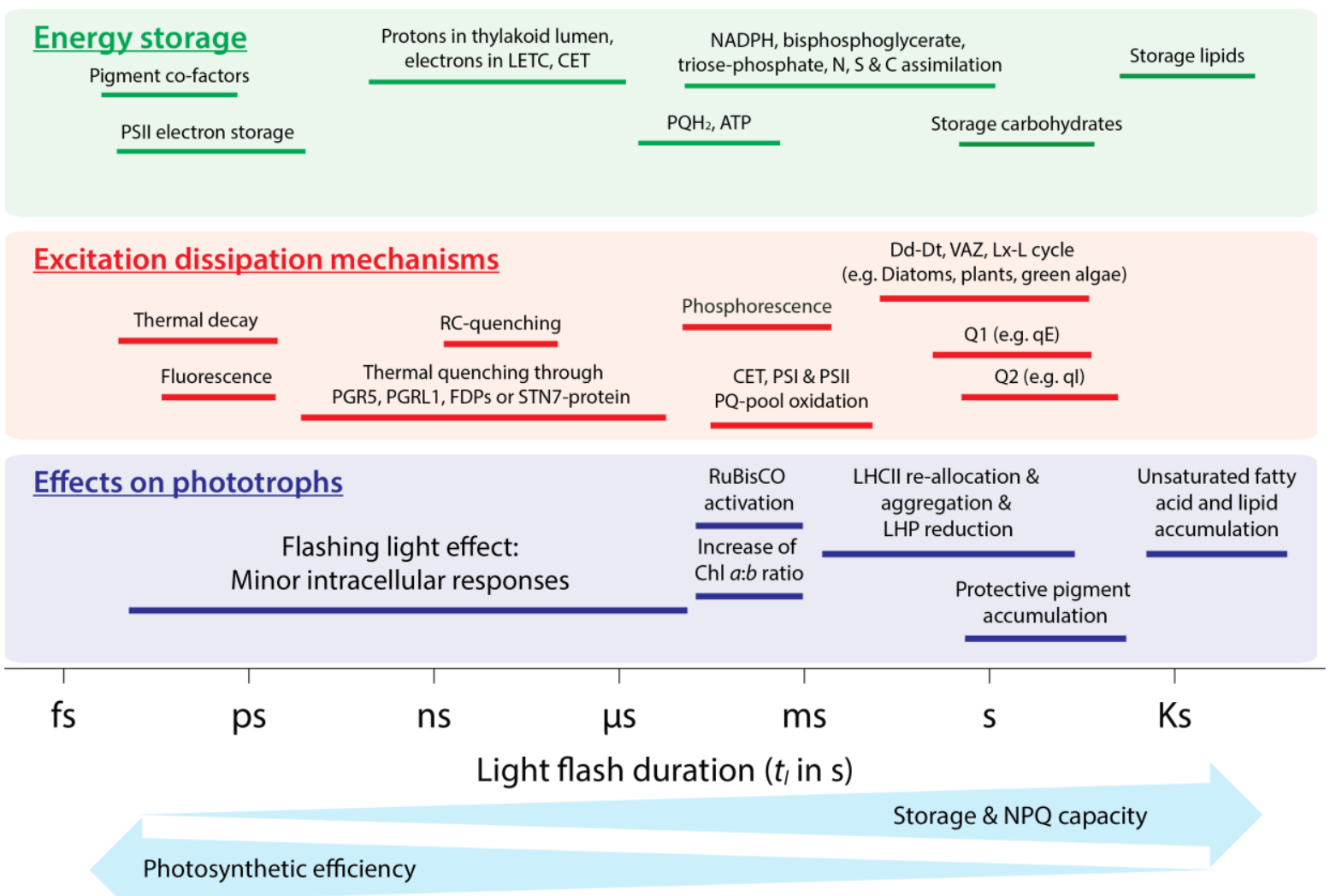

Figure 2 - Response time scales of phototrophs exposed to different flash period durations

$143\left(t_{l}\right)$ with a high intense flash intensity $\left(I_{l}\right)$ that arise if flashing light composed of a short constant

144 duty cycle (e.g., $\varnothing<0.1)$ and a saturating averaged light intensity $\left(I_{a}\right)$ is used. A given flash

145 period duration is inversed proportional to the flashing light frequency $(f)$. Time scales of events

146 were obtained from results summarised in Tables 1 and $S 1$ and elsewhere [33, 34, 37]. Bar

147 length represents the approximate time scale of the initiation of a given event. Abbreviations:

148 CET, Cyclic Electron Transfer; Chl, Chlorophyll; Dd-Dt, diadinoxanthin-diatoxanthin; FDPs,

149 flavodiiron proteins; LET, linear electron transfer chain; LHC, light harvesting complex; LHP,

150 light harvesting pigment; Lx-L, lutein epoxide; PGR5, proton gradient regulator 5; PGRL1, a

151 ferredoxin-plastoquinone reductase that is apparently involved in CET in chlorophytes; PQ,

152 plastoquinone; $\mathrm{PQH}_{2}$, plastoquinol; $\mathrm{PS}$, photosystem; Q1/2, quenching sites 1 and 2; qE, energy

153 state quenching; qI, photoinactivation of photosystem II; RC, reaction centre; RuBisCO,

154 ribulose-1,5-bisphosphate-carboxylase/oxygenase; STN7, Serine/threonine-protein kinase

155 involved in the adaptation to changing light conditions; VAZ, violaxanthin-astaxanthin-

156 zeaxanthin.

A medium-term energy storage (ns- $\mu$ s time scale) may take place via components and products of the non-cyclic photosynthetic electron transfer chain. Examples are the plastoquinone bound to PSII $\left(\mathrm{Q}^{-}\right)$, plastoquinol $\left(\mathrm{PQH}_{2}\right)$ in the plastoquinone (PQ) pool [34], 
161 protons in the stroma coming from the water-splitting reaction catalysed by the water oxidising complex and ATP produced by the ATP synthase in the thylakoid [35]. However, if the previous storage mechanisms are unable to handle excess energy, other non-photochemically quenching reactions and biomolecules seem to play a protective role: e.g. Mehler-like reactions, the proton gradient regulator PGR5, the ferredoxin-plastoquinone reductase PGRL1, the serine/threonine-protein kinase STN7, and several flavodiiron proteins [8, 36-38].

For long-term energy storage (ms-s time scale), phototrophs produce reduced equivalents (e.g. NADPH) or "high energy" chemical bonds via ATP-dependent nitrogen and sulphur assimilation as well as carbon fixation. The last process yields Calvin-Benson cycle intermediates containing ATP-dependent high energy bonds, such as bisphosphoglycerate or triose-phosphate [30]. At this time scale, excess energy can be quenched via re-oxidation of the PQ pool through the quinol terminal oxidase, phosphorescence or through the initiation of diadinoxanthin-diatoxanthin, violaxanthin-astaxanthin-zeaxanthin or lutein epoxide cycles [3941].

Even longer-term energy storage is possible. Biochemical processes at time scales of minutes and hours, such as the accumulation of non-structural low molecular weight sugars, starch or amino acids (e.g. glutamine as the first amino acid resulting from nitrogen assimilation) can be used to store energy [42]. Under these conditions, excess energy can be quenched through the same photoprotective pigment cycles as under ms-s conditions, but also through high-energy-state (qE) quenching and photoinactivation of PSII (here referred to as qI; $[33,41,43])$. In order to decrease excess energy that phototrophs receive under long-lasting light periods, reversible phosphorylation of the light harvesting complex II and decrease in the light harvesting antenna size might occur [37, 39].

Generally, the ratio between non-photochemically and photochemically quenched energy, and the probability of damaging the photosystems by ROS evolution, due to a failure of the excitation dissipation mechanisms in place, increases with light flash period duration, causing a drop in photosynthetic efficiency. Under frequencies and duty cycles that are too low and too short, respectively, for obtaining a biological flashing light effect, phototrophs use more complex and energy demanding excitation dissipation mechanisms during the light period (e.g. photoprotective pigment synthesis or high-energy-state quenching [33]) and respiration rates exceed photosynthetic rates during the extended dark period [15, 44, 45]. Both situations will ultimately decrease or restrict net photosynthetic efficiency [27] and alter the biochemical profile and appearance of microalgal and cyanobacterial cells. Changes include cell size, pigment composition, intracellular ultrastructure, expression of protective proteins (e.g. PGR5 
195 or STN7), the ratio between PSI and PSII [8], light harvesting antenna size, ribulose-1,5-

196 bisphosphate-carboxylase/oxygenase (RuBisCO) activity, or sugar and starch contents [8, 11,

197 30, 46-51]. These changes are typical for responses of microalgae to intense light $[49,50]$ and

198 thus can be used as indicators if the frequency and duty cycle are inadequate for a phototroph

199 to experience the biological flashing light effect. Conversely, if phototrophs are exposed to

200 increasing frequencies (e.g. $f>10 \mathrm{~Hz} ; \varnothing \approx 0.1-0.5$ ), these changes become less obvious [46-48,

201 52, 53], because a phototroph buffers and quenches photoenergy delivered during the light

202 period with a similar efficiency to that under continuous light. Nevertheless, lower intracellular

203 chlorophyll $a$ and carotenoid contents are probably not good indicators for the flashing light

204 effect, as lower amounts of these pigments occur in several species under a wide range of

205 flashing light conditions (e.g. $f=0.1-100 \mathrm{~Hz}$; Table 1; [44, 46-49]). 
Table 1 - Impacts of flashing light with different duty cycles and frequencies on microalgal composition. Refer to Table S1 (supplementary data) for a detailed 208 overview of relevant flashing light studies on microalgae, cyanobacteria and plants.

\begin{tabular}{|c|c|c|c|c|}
\hline Microalga & $\begin{array}{c}\text { Frequencies } \\
(f)\end{array}$ & $\begin{array}{c}\text { Duty cycles } \\
(\varnothing)\end{array}$ & Outcome & $F$ \\
\hline $\begin{array}{l}\text { Chlamydomonas } \\
\text { reinhardtii }\end{array}$ & $1-100 \mathrm{~Hz}$ & 0.5 & $\begin{array}{l}\text { Absorption spectra unaffected by flashing light, no obvious shift in carotenoid:chlorophyll } \\
\text { ratio in absorption spectra. }\end{array}$ & {$[20,2$} \\
\hline $\begin{array}{l}\text { Chlamydomonas } \\
\text { reinhardtii }\end{array}$ & $0.5-5 \mathrm{~Hz}$ & 0.5 & Fatty acid profile and total lipids were mostly unaffected by flashing light. & {$[54]$} \\
\hline Chlorella kessleri & $5 \mathrm{~Hz}-37 \mathrm{KHz}$ & 0.5 & $\begin{array}{l}\text { Higher intracellular chlorophyll concentrations under flashing light as compared to continuous } \\
\text { light. }\end{array}$ & [11] \\
\hline Chlorella pyrenoidosa & $\begin{array}{c}2.5- \\
25 \mathrm{KHz}\end{array}$ & $0.0125-0.125$ & $\begin{array}{l}\text { Immediate sugar accumulation when exposed to saturating light flashes for } 18 \mathrm{~h} \text {. Dark periods } \\
\text { lasting only } 6 \mathrm{~h} \text { led in turn to an accumulation of nucleic acids and a complete consumption of } \\
\text { accumulated sugars. Protein and chlorophyll levels unaffected. }\end{array}$ & [24] \\
\hline $\begin{array}{l}\text { Haematococcus } \\
\text { pluvialis }\end{array}$ & $25-200 \mathrm{~Hz}$ & $\begin{array}{l}0.17,0.33 \\
0.67\end{array}$ & $\begin{array}{l}\text { Final astaxanthin and biomass concentration in the medium was higher under flashing light as } \\
\text { compared to continuous light. With increasing duty cycle but same frequency, the final } \\
\text { volumetric astaxanthin concentration rose. The use of flashing light lowered the energy } \\
\text { consumption for astaxanthin production by up to } 70 \% \text {. }\end{array}$ & {$[55]$} \\
\hline Isochrysis galbana & $10 \mathrm{KHz}$ & 0.5 & $\begin{array}{l}\text { No effects on total lipid content in I. galbana or cell weight. Fatty acid profile was similar } \\
\text { under flashing and continuous light. }\end{array}$ & {$[52]$} \\
\hline $\begin{array}{l}\text { Nannochloropsis } \\
\text { oceanica CY2 }\end{array}$ & $7,8,9 \mathrm{~Hz}$ & 0.5 & No significant differences in EPA content between cells under flashing and continuous light. & {$[53]$} \\
\hline $\begin{array}{l}\text { Nannochloropsis } \\
\text { salina }\end{array}$ & $1-30 \mathrm{~Hz}$ & $0.1,0.33$ & $\begin{array}{l}\text { Flashing light had no effect on total lipid content and caused usually lower accumulation of } \\
\text { chlorophyll } a \text { and carotenoid:chlorophyll ratios (Except } f=10 \mathrm{~Hz}, \varnothing=0.33 \text { ). }\end{array}$ & [48] \\
\hline
\end{tabular}


Porphyridium

purpureum

$0.17-100 \mathrm{~Hz}$

$0.17,0.5$

Scenedesmus

bicellularis

$\sim 100 \mathrm{~Hz}$

0.5

Scenedesmus obliquus

$5,10,15 \mathrm{~Hz}$

Chlamydomonas

reinhardtii

$0.00138-1 \mathrm{~Hz}$

0.5

Dunaliella salina

$0.017-5 \mathrm{~Hz}$

$0.4-0.66$
The frequency of $0.17 \mathrm{~Hz}$ increased the intracellular phycoerythrin and chlorophyll $a$ content as compared to continuous light and $25 \mathrm{~Hz}$ and $\varnothing=0.33$. Bound and free polysaccharides were affected marginally. Production rates of phycoerythrin and free polysaccharides were highest under $f=100 \mathrm{~Hz}, \varnothing=0.5\left(I_{l}=540 \mu \mathrm{mol}\right.$ photons $\left.\mathrm{m}^{-2} \mathrm{~s}^{-1}\right)$.

The long-term exposure to flashing light did not affect total lipids, proteins, carbohydrates, fatty acids and amino acids. However, flashing light lowered slightly chlorophyll $a$ and $b$ levels, increased chlorophyll $a / b$ ratios, decreased carotenoid contents and increased carotenoid/chlorophyll $a$ ratio. RuBisCO initial activity (not activated) and RuBisCO total [47] activity (activated) were significant higher only under flashing light at a moderate irradiance of $I_{a}=175 \mu \mathrm{mol}$ photons $\mathrm{m}^{-2} \mathrm{~s}^{-1}$, whereas low $\left(I_{a}=87.5 \mu \mathrm{mol}\right.$ photons $\left.\mathrm{m}^{-2} \mathrm{~s}^{-1}\right)$ and high $\left(I_{a}=350 \mu \mathrm{mol}\right.$ photons $\left.\mathrm{m}^{-2} \mathrm{~s}^{-1}\right)$ averaged light intensities had no effect.

Carotenoid:chlorophyll ratio and chlorophyll $a$ content in cells were lower under flashing light. Carbohydrate, lipid, and protein contents were unaffected.

Decreasing chlorophyll $a$ content with increasing frequency $\left(I_{a}=220 \mu\right.$ mol photons $\left.\mathrm{m}^{-2} \mathrm{~s}^{-1}\right)$. Lowest amount of chlorophyll $a, b$ and carotenoids under $f=1 \mathrm{~Hz}$.

Usually lower chlorophyll $a$ content under flashing light $\left(I_{a}=400 \mu \mathrm{mol}\right.$ photons $\left.\mathrm{m}^{-2} \mathrm{~s}^{-1}\right)$ as compared to continuous light. A $f=5 \mathrm{~Hz}$ showed similar results as compared to continuous [50] light. D. salina exposed to flashing light conditions showed always lower total lipid content. 
Emerson and Arnold [57] demonstrated that a short light period with an adequate flash intensity can excite all "open" reaction centres, whereas a sufficient long dark period allows all reaction centres to "re-open" and harvest most of the incoming photons of the next light flash. A later study by Radmer and Kok [58] quantified that a light harvesting complex containing 400 chlorophyll molecules harvest 2,000 electrons per second under full sunlight, whereas the subsequent carbon fixation reactions are able to process only 100-200 electrons per second. They showed that the photosynthetic apparatus could only use a small portion of incident light under continuous light, while, most of the time, reaction centres are closed and light is nonphotochemically quenched.

Recent findings about excitation dissipation mechanisms and the quenching role of the PQ pool may allow other approaches to determine the optimal flashing light settings required for photosynthesis. It has been suggested that the species-dependent storage capacity of the PQ pool for plastoquinol may define the threshold frequency, duty cycle and the required averaged light intensity beyond which the flashing light effect occurs [26, 59]. Vejrazka and colleagues [21] and Hüner et al. [34] pointed out that if excess plastoquinol is generated, the plastoquinol:plastoquinone $\left(\mathrm{PQH}_{2}: \mathrm{PQ}\right)$ ratio becomes too high, leading to over-reduction of the PQ pool, which will prevent the reaction centre II from re-opening [34]. Such over-reduction occurs if transfer rates of photonic energy exceed the kinetics of its use by metabolic pathways that promote growth, including those involved in nitrogen, sulphur and carbon utilization. Detrimentally, excess energy can lead to photoinhibition and photo-oxidative damage due to ROS evolution. Phototrophs can dissipate this energy by, for example, (i) activating the cyclic electron flow, (ii) phosphorylating and migrating the light harvesting complex II towards PSI to reinforce the cyclic electron transfer used to oxidize the PQ pool or (iii) activating the xanthophyll cycle [39]. However, to avoid photoprotective mechanisms and thus inefficient photonic energy usage, light supply should take place in balance with the reaction kinetics of the linear electron transfer chain. Interestingly, a light flash lasting picoseconds is already long enough to excite and close the PSII reaction centres [26]. To restore the ground state of PSII, and thus to re-open the reaction centre II, requires the transfer of this charge from the PSII to the PQ pool in the form of plastoquinol [60] and the reduction of PSII through the water oxidising complex. In order to avoid excess of plastoquinol reducing equivalents in the PQpool, the reduction rate of PQ to plastoquinol at the $\mathrm{Q}_{b}$ site of PSII should be similar to the plastoquinol oxidation rate at the $\mathrm{Q}_{0}$ site of the cytochrome $b_{6} f$ complex. However, an imbalance easily arises because the oxidation of one plastoquinol takes longer (ca. $\approx 3-5 \mathrm{~ms}$ ) than reducing 
PSII by the water oxidising complex $(\approx 1-3 \mathrm{~ms})$. To mitigate such imbalances in the linear electron transfer chain, flashing light may be tailored to a flash period duration of a few hundred picoseconds to reduce efficiently the reaction centre II without triggering excitation dissipation mechanisms, and a dark period of 3-5 ms to allow the timely oxidation of plastoquinol, avoiding the overreduction of the PQ pool.

However, this kinetics would correspond to a duty cycle of only $\phi \approx 10^{-8}$ and a frequency of $f \approx 300-500 \mathrm{~Hz}$. Such settings require low switching times, which are problematic to implement with the current technologies available (Box 2). Indeed, frequencies higher than $300 \mathrm{~Hz}$ did usually result in a flashing light effect in most phototrophs if the averaged light intensity was near saturation (e.g., $I_{a} \geq 100 \mu \mathrm{mol}$ photos $\mathrm{s}^{-1} \mathrm{~m}^{-2}$; Table S1). However, decreasing the averaged light intensity towards sub-saturating levels appears to require higher frequencies to obtain the flashing light effect $[26,61]$. More specifically, Martín-Girela et al. [61] found a $\mathrm{CO}_{2}$ fixation efficiency of 6.2 photons per fixed $\mathrm{CO}_{2}$ molecule, which was beyond theoretical limits $\left(\sim 8\right.$ photons $\left.\mathrm{CO}_{2}^{-1}\right)$ at a frequency of $10,000 \mathrm{~Hz}(\varnothing=0.05)$ with an averaged light intensity that was only $5 \%$ of the photosaturating intensity.

On the other hand, it remains to be seen how phototrophs respond if they are exposed to extremely low duty cycles (e.g. $\phi<10^{-8}$ ), with a flash intensity $\approx 10^{8}$ times higher than the averaged light intensity, which corresponds also to a photon penetration depth 8 times higher as compared to that of continuous lighting [7]. Under such timescale, dissipation of excess energy may only take place via fluorescence or thermal decay, and other more complex and energy-demanding quenching mechanisms (e.g. mediated by PGR5, PGRL1, flavodiiron proteins or STN7) cannot be activated in time [37,62]. This may lead to a more efficient light utilisation and higher photosynthetic efficiencies. On the other hand, these conditions could also increase flash intensities above a threshold that would instantly cause photodamage. If so, an under-saturating averaged light intensity could be sufficient to achieve the same or even enhanced photosynthetic rates compared to continuous lighting with a saturating light intensity, leading to lower power consumption of artificial lighting. Notably, flash intensities that are inhibitory if emitted continuously do not inhibit the phototroph if the frequency is high and duty cycle is short enough (e.g. $f>1 \mathrm{KHz}, \varnothing<0.1$ ) for a given averaged light intensity [24-26, 6365]. For example, Tennessen et al. [26] exposed tomato leaves to photoinhibitory flash intensities $\left(I_{f}=5000 \mu \mathrm{mol} \mathrm{s}^{-1} \mathrm{~m}^{-2}\right)$ at a short duty cycle $(\varnothing=0.01)$ and a high frequency $(f=5000 \mathrm{~Hz})$ without impairing photosynthesis. Nevertheless, Ley and Mauzerall [66] found that flash intensities higher than 22,000-37,000 $\mu$ mol photons $\mathrm{m}^{-2} \mathrm{~s}^{-1}$ (e.g., $10^{16}$ photons $\mathrm{cm}^{-2}$ supplied during light periods of 450-750 ns) can indeed cause a decline of oxygen evolution 
rates in Chlorella vulgaris cultures $\left(t_{d}=2 \mathrm{~s}\right)$. These findings indicate that averaged light 281 intensity, flash intensity, frequency and duty cycle are interdependent and must be well balanced to reap the benefits of artificial flashing light-based phototrophic cultivation.

\section{Physical boundaries}

The most important physical factor of flashing light is the potential to mitigate light attenuation and increase light delivery in concentrated microalgal cultures [13, 49, 64, 67, 68]. Current efforts to enhance light delivery include intensive mixing, light path minimization, antenna size reduction, waveband tailoring or inclusion of fibres and nanoparticles as waveguides into the photobioreactor [9, 69-72]. In addition to these approaches, high light intensities can increase photon penetration depth in suspensions as defined by Beer-Lambert's law [7]. The Beer-Lambert's law [7] describes a linear increasing light penetration depth into microalgal cultures with exponentially rising light intensity, though effects by fluorescence or light scattering by different algae are not considered.

Too high light intensities cause photoinhibition of microalgae at the periphery of the photobioreactor, an effect that is mitigated by increasing mixing rates. Higher mixing rates minimise retention time of cells in the high light zone near the walls and in the dark zones in the middle of the reactor. These high mixing rates improve light and can provide light-dark cycles for cells fast enough to obtain the flashing light effect [13, 14]. Particularly high productive cultivation systems benefit from high culture concentrations and light intensities [73]. However, these conditions require extreme high mixing velocities, resulting in high energy consumption [44, 65, 74], shearing, cavitation and pressure changes that impair the physiology and viability of the microalgal cell [73]. Alternatively, a light source can directly emit flashing light, which allows the generation of intense light flashes at frequencies and low duty cycles that do not occur in nature or in any culturing vessel just by adjusting the mixing velocity of the growth medium.

As mitigating light attenuation is one of the main arguments for flashing light-induced growth enhancement $[13,49,64,67,68]$, production systems that operate at high cell concentrations or culturing vessels with long light path lengths are promising targets for flashing light-related power savings. Although such trend has only be observed among few studies $[11,64,73]$, the true potential of mitigating light attenuation in dense microalgal cultures remains uncertain, particularly at extreme high light flash intensities (e.g. $I_{f}>10,000 \mu \mathrm{mol}$ photons $\left.\mathrm{m}^{-2} \mathrm{~s}^{-1}\right)$, delivered at low duty cycles (e.g., $\varnothing<0.01$ ) and high frequencies (e.g. $f>1 \mathrm{KHz}$ ). 
315 In artificial light-based microalgal production, light with low and high light periods (e.g., 316 flickering or fluctuating light) is naturally emitted by common gas discharge lamps, whereas flashing light is generated when light intensity of LEDs is controlled via pulse-width modulation (e.g., Fig. 1). The intensity of light emitted by fluorescent lamps and mercury or sodium vapour lamps changes between maximum and minimum values (often referred as "flickering light") at a ballast- and grid-dependent frequency [47]. For example, fluorescent lamps driven by a conventional magnetic or electronic ballast emit flickering light at a frequency of $100-120 \mathrm{~Hz}$ and $40-120 \mathrm{KHz}$, respectively. On the other hand, induction lamps operate at frequencies from hundreds of $\mathrm{KHz}$ to tens of $\mathrm{MHz}$ [75]. Therefore, it becomes clear that neither induction nor gas discharge lamps should be used as "non-flashing" controls in any flashing light study. In addition, gas discharge lamps are inefficient in terms of photon conversion efficiency if operated at low duty cycles and may be unsuitable for customised flashing light modulation. Alternatively, amplitude or pulse-width modulated LEDs can efficiently emit continuous and flashing light, respectively. However, commonly available pulse-width modulated dimmers generate flashing light only between frequencies of 150 and $300 \mathrm{~Hz}$, which may be not sufficient to obtain a flashing light effect if dimmed, though higher frequencies and lower switching times are possible (Box 2). For microalgal production, a promising flashing LED device may operate at a (sub-)saturating averaged light intensity, which requires a light flash intensity that increases in inverse proportion to the duty cycle. The emission of high light flash intensities is possible if the stock densities of LEDs in a luminary array is increased, which has additional costs. Alternatively, the number of photons emitted per light emitter can be enhanced under flashing light if the forward current to an LED is increased far beyond the nominal currents used under continuous operating conditions. This so-called "overloading" demands precise switching regimes to extract the highest number of photons with the highest efficiency possible. Considering all losses between power source and light emission of a flashing light system (referred to as wall plug efficiency), most discriminative parameters include i) response time and photon conversion efficiency of the LED; ii) operating frequency and duty cycle; and iii) the efficiency factor of the electronic ballast and pulse-width modulation unit. Generally, the wall plug efficiency of a flashing lighting system decreases with increasing frequency, decreasing duty cycle and increasing forward current due to working and switching losses at transistors and LEDs [76]. The efficiency drop can be damped if transistors and LEDs display low response times, low working and switching losses under the used flashing light condition and currents. A joint effort between physicists and biologists is 
thus necessary for the development of efficient flashing light systems that enhance energy use in artificial light-based microalgal production.

\section{Concluding Remarks and Future Perspectives}

Using flashing lights is a promising strategy to supply photonic energy to phototrophic organisms, increasing biomass productivities and reducing the power consumption in artificial light-based production systems. Flashing light can also be applied to established methods used to improve the photosynthetic performance of microalgal cultures such as mixing, light guides or waveband tailoring. The optimal settings of a flashing light regime should correspond to a frequency high enough to obtain the same or higher photosynthetic efficiencies than those under continuous light (e.g., $f>300-500 \mathrm{~Hz}$; biological factor) at a shortest possible duty cycle to obtain the highest possible photon penetration depth (physical factor), but both within the range of adequate power consumption (technical factor). So far, most studies have tested flashing light conditions with low frequencies $(f<100 \mathrm{~Hz})$ and relatively high duty cycles $(\varnothing>0.1)$ to mimic conditions that are present in mixed microalgal cultures. However, data beyond these conditions will be of particular interest for artificial light-based microalgal production. Mainstream flashing light research and industrial application benefit specifically from inexpensive and technically mature LED modules as light sources. Nevertheless, current LED technology cannot modulate flashing light in the response time scales close to the boundaries imposed by the biological responses, such as light harvesting events within the range of femtoto picoseconds. A possible solution to this limitation is the use of faster responding laser diodes (see Outstanding Questions), which could replace common LEDs in cutting-edge research, as well as in future industrial production facilities. Cultures exposed to sub-nanosecond light flashes might obey the laws of quantum mechanics, which can result in unforeseeable effects on photosynthesis and growth of phototrophs. Research on charge transfer on quantum level as e.g. implemented by the EU project H2020-MSCA-QuantumPhotosynth may shed new light on the limits of photosynthesis and more efficient photon utilisation by microalgae

\section{Acknowledgements}

This study was funded by the Foundation for Science and Technology (FCT, Portugal; CCMAR/Multi/04326/2013), Nord University, Nordland County Government (project Bioteknologi- en framtidsrettet næring), and ALGARED+ 1398 EP - INTERREG V-A España- 
380 University and the Portuguese Foundation for Science and Technology 381 (SFRH/BD/105541/2014), respectively. 


\section{Box 1 - Kinetics of the linear electron transfer chain (LET)}

383

Photons coming from a light source are absorbed through light harvesting pigment complexes within femtoseconds. About 300-500 ps are needed to transfer shared excited energy states (excitons) through inter-protein hopping and magnetic resonance to the reaction centres of PSII (reaction centre II or P680), causing the excitation of an electron. The reaction centre requires two electrons for reduction and "closure" (i.e. P680"; Fig. I; [32]). Once the reaction centre is in the "closed" state, further excess photon energy cannot be transferred to the reaction centre II and is released through energy dissipation mechanisms. The low redox state of the P680* reduces the primary electron acceptor pheophytin within 3 to $8 \mathrm{ps,} \mathrm{becoming} \mathrm{oxidised}$ $\left(\mathrm{P}_{680}\right)$. The electrons from pheophytin are transferred to the primary $\left(\mathrm{Q}_{\mathrm{a}}\right)$ and secondary $\left(\mathrm{Q}_{\mathrm{b}}\right)$ acceptor sites within $\sim 200-500 \mathrm{ps}$ and 700-1200 $\mu$ s, respectively. Upon $\mathrm{Q}_{\mathrm{b}}$ reduction, this site acquires protons from the stroma, forming plastoquinol. In turn, plastoquinol diffuses towards the PQ pool in the thylakoid membrane upon exchange with one PQ molecule, which binds to the $\mathrm{Q}_{\mathrm{b}}$ site. The PQ pool serves as energy store. The energy is retrieved upon the oxidation of plastoquinol by the cytochrome $b_{6} f$ complex via the q-cycle [77].

The high redox potential of $\mathrm{P} 680^{+}$initiates an electron transfer from the water oxidising complex through the intermediate electron carrier tyrosine, which reduces $\mathrm{P}_{680}{ }^{+}$in a succession of steps (S0-S3). Full oxidation of two water molecules and the release of four electrons takes place in about 1-2 ms [35]. As P680 is formed, the reaction centre II re-opens and the subsequent exciton capture takes place.

The slowest ( $3-5 \mathrm{~ms})$ and thus limiting step in the linear electron transfer chain is the oxidation of plastoquinol by the cytochrome $b_{6} f$. Two protons are released into the thylakoid lumen and electrons are transferred towards plastocyanin. Plastocyanin transfers electron towards PSI within 150-550 $\mu$ s. In PSI, electrons are passed to the electron donor P700 (reaction centre I), forming $\mathrm{P} 700^{*}$ through photon energy delivered by the light harvesting complex I within femtoseconds. Electrons are passed to the electron acceptors $A_{0}, A_{1}$ and the $4 \mathrm{Fe}-4 \mathrm{~S}$ iron sulphur centres $\mathrm{Fx}, \mathrm{Fa}$ and $\mathrm{Fb}$ within picoseconds, reducing the final electron acceptor, ferredoxin. Because of these short turnover times, the reactions in P700 are considered to be a spontaneous reaction $[32,34]$. Ferredoxin can donate electrons to ferredoxin-NADP(+) reductase to form NADPH, completing the LET. The cyclic electron transfer chain is activated to produce additional ATP and NAPDH required for carbon assimilation via the Calvin-Benson cycle. 
$\mathrm{N} \& \mathrm{~S}$ assimilation $\mathrm{H}_{2} \mathrm{O}_{2}$ - Peroxiredoxins Thioredoxins

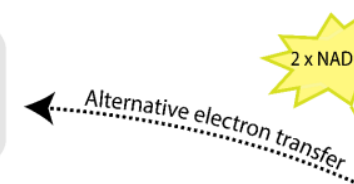

(H)

(H)

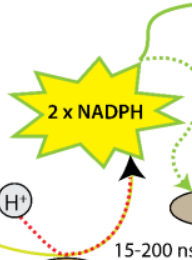

$\mathrm{ns}$
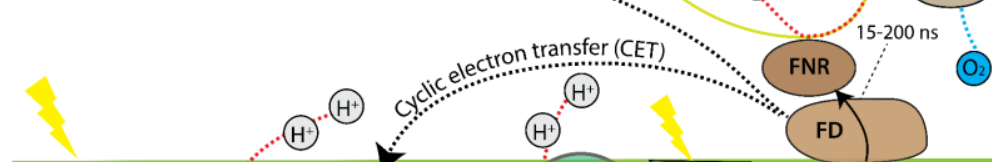

FNR

FD

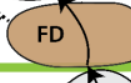

.

Stroma (outside)

Thylakoid membrane

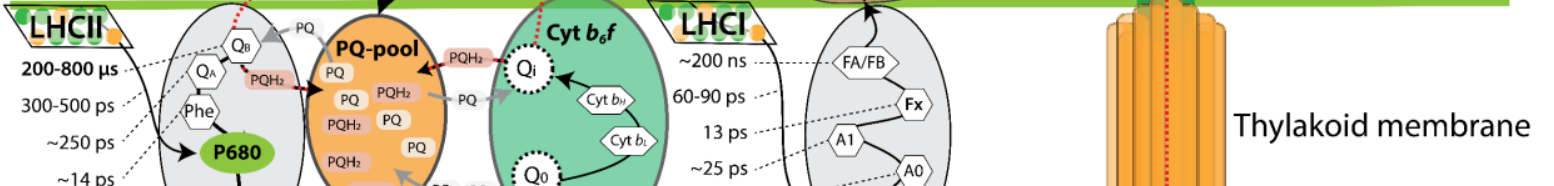

(H)( (A)

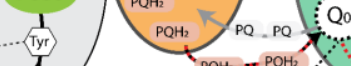

3-5 ms

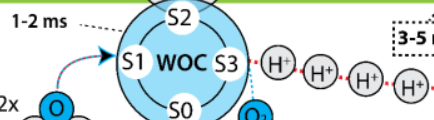

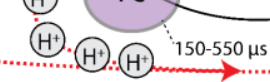

Lumen (inside)

Figure I - Simplified diagram about the major kinetics of electron transfers in phototrophs, where the linear electron transfer chain (LET) is depicted and other alternative pathways are indicated. Reaction times and pathways were summarized from Ref [35, 39]. Note that stoichiometric values for $\mathrm{H}+, \mathrm{ATP}$ and NADPH are variable. To balance electron flow under fluctuating light regimes, phototrophs use species dependently different Flv proteins to reduce oxygen to water at the expense of NADPH or electrons from the photosystems [85]. Abbreviations: $A_{1}$, phylloquinone-based electron acceptor; ADP, adenosine diphosphate; $A_{0}$, chlorophyll based electron acceptor; ATP, adenosine triphosphate; Cyt $b_{6} f$, cytochrome $b_{6} f$ complex; Cyt $b_{L / H}, f$, b-type hemes cytochrome $b_{L / H}, f ; \mathrm{FA} / \mathrm{FB} \& \mathrm{Fx}$, electron acceptors $4 \mathrm{Fe}-4 \mathrm{~S}$ irons sulphur centres; FeS, Rieske iron-sulphur protein; FD, ferredoxin; Flv, flavodiiron protein; FNR, Ferredoxin-NADP(+) reductase; LHC, light harvesting complex; NADP+, nicotinamide adenine dinucleotide phosphate; NADPH, nicotinamide adenine dinucleotide phosphate (reduced); P680, Photosystem II; P700, Photosystem I; PC, plastocyanin; Phe, pheophytin; Pi, inorganic phosphorus; $\mathrm{PQ}$, plastoquinone; $\mathrm{PQH}_{2}$, plastoquinol; $\mathrm{Q}_{\mathrm{A}}$, primary acceptor site; $\mathrm{Q}_{\mathrm{B}}$, secondary acceptor site; $\mathrm{Q}_{\mathrm{i}}$, quinone reductase; $\mathrm{Q}_{\mathrm{o}}$, quinol oxidase; $\mathrm{RuBP}$, ribulose-1,5-bisphosphate; Tyr, tyrosine; WOC, Water oxidising complex. 


\section{Box 2 - Technical limits of flashing light sources}

The efficiency of a flashing light system depends on working and switching losses at transistors built in pulse-width modulators, controlling units and LEDs that interact differently with applied current, frequency and duty cycle. For example, work losses in transistors switches increase if duty cycle and currents are high, but they are frequency-independent. Switching losses increase with frequency, though they are current-independent [76]. Photon extraction potential from LEDs is higher if forward current is increased (i.e. LED overloading), and light and dark periods are short and long enough, respectively, to allow sufficient heat dissipation from the LED-chip (Fig. II). In this case, frequency and duty cycle are interdependent.

Overloading is a valuable option for flashing light applications, permitting the operation of an LED under higher currents that exceed nominal levels (e.g., by increasing the supplied voltage), resulting in maximal photon flux during the duty cycle and heat dissipation during the dark period [11, 24]. Through overloading, the LED operates at current densities beyond which the "droop effect" occurs. As result, the photon conversion efficiency drops with increasing forward currents and maximal photon extraction cannot exceed a given threshold [78]. During overloading and shortening duty cycle, the maximal extractable averaged light intensity $\left(I_{a}\right)$ per LED decreases, but the maximal applicable instantaneous forward current and extractable flash intensity increases.

Another parameter is the LED minimal response time $\left(t_{r_{-} \min }\right)$, which is the major cause for electrical losses when emitting flashing light. Here, the depletion region as central internal element of any LED chip creates capacitances that limit the response of single-coloured LEDs to a few nanoseconds ( $t_{r_{-} \min } \approx 1-50 \mathrm{~ns}$ ). Note that organic LEDs display high capacitances while phosphor-converted LEDs have long (electro-)luminescence decay times, which restricts $t_{r_{-} \min }$ to approximately $\geq 1 \mu \mathrm{s}$ [79-81]. Standard laser diodes display very low capacitance, allowing $t_{r_{-} \min }<500 \mathrm{ps}$ [82]. Overloading and chip size increases these capacitances and thus response times, limiting maximal adjustable frequencies or duty cycles. Lowering response times of (organic) LEDs is an active field of research as required for efficient visible light communication or screens [80, 82,83].

Broadband flashing light research at nanosecond scales can use widely available signal generators connected to high power single-coloured LEDs or laser diodes as a cheap solution (e.g. $<2000 €$ ). For larger scale systems with high light output, a more robust and cheaper system may be used, based on a slower responding open source system ( $\geq 1 \mu \mathrm{s})$ consisting of an Arduino microcontroller coupled with standard LED luminaries (for examples see [50, 84]). 
A

466

467

468

469

470

471

472

473

474

475

476

477

478

479

480

481

482
B

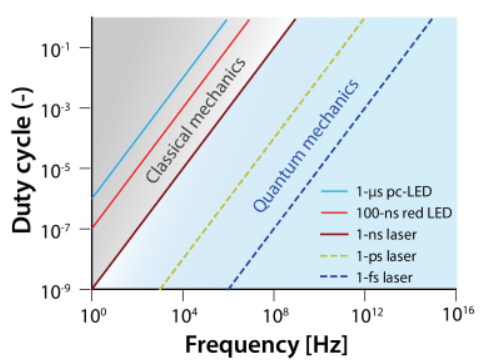

C

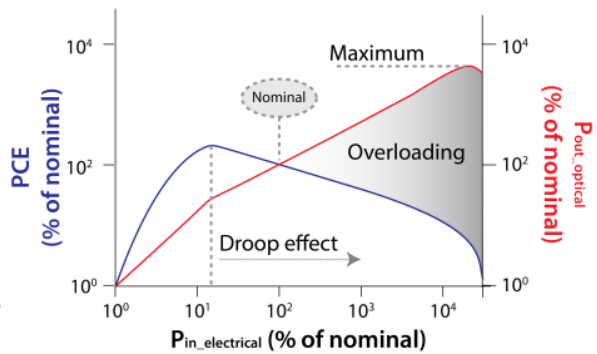

Figure II - The efficiency of LEDs or laser diodes under pulsed power supply can be calculated from frequency response graphs, called bode plots (A). Here, the cut-off frequency $\left(f_{c}\right)$ refers to the maximal adjustable frequency with maximal $50 \%$ power losses (commonly referred to as $3 \mathrm{~dB}$ point). From $f_{c}$, response times and possible adjustable flashing light regimes can be calculated for different light sources (B). If switching regimes are below $1 \mathrm{~ns}$, a shift from laws of classical to quantum mechanics occurs. Overloading $(C)$ is achieved if the input power $\left(P_{\text {in_electrical }}\right)$ increases due to higher forward currents that exceed nominal levels. Nominal conditions are obtained when LEDs operate under continuous power supply and rated (or nominal-) currents at a given temperature. Under a nominal power input ( $P_{\text {in_electical }}$ ), an LED achieves its nominal light output $\left(P_{\text {out_optical }}\right)$ and nominal efficiency (e.g., photon conversion efficiency; PCE $=P_{\text {out_optical }}\left(P_{\text {in_electrical }}\right)$ as defined by the manufacturer. LED light output comes at the cost of efficiency after passing a critical current density beyond which the droop effect occurs (dashed line) and usually reaches a peak (maximum) with subsequent decline. All values given are examples and may differ among diodes. Abbreviations: pc-LED: phosphor converted LED, AlGaAs LED: gallium-aluminium-arsenide LED. 


\section{Glossary box}

- Intermittent light includes flashing or pulsed light and fluctuating, flickering or oscillating light. Light and dark periods of flashing or pulsed light conditions shift in an all-or-nothing, rectangular waveform. Fluctuating or oscillating light is a fluent transition between high and low light periods, whereas instantaneous light intensities alter continuously over time, following usually a sinusoidal waveform. Sunflecks or cells moving from light to dark zones within a photobioreactor trough mixing usually follow fluctuating light patterns.

- Light attenuation: Self-shading by microalgal cells is the most challenging bottleneck limiting the productivity and maximal achievable cell concentration in photobioreactors. Cells located at the periphery of a culture absorb most of the incoming light and may become photoinhibited, whereas cells at the inner layers remain in the dark and become photolimited. This results in high respiration and energy dissipation rates, causing inefficient photobioreactor use. The depth of the light penetration depends primarily on absorption by cells under cultivation, incoming light intensity and wavelength, cell morphology (e.g. cell size) and biochemistry (e.g. pigment contents).

- Mehler and Mehler-like reactions are controlling light-depended $\mathrm{O}_{2}$ consumption. Unlike the Mehler reaction, the Mehler-like reaction involves flavodiiron proteins to reduce $\mathrm{O}_{2}$ without $\mathrm{ROS}$ evolution. Mehler-like reactions enable cyanobacteria, microalgae, and plants to cope efficiently with intermittent light regimes.

- Minimal response times $\left(t_{r_{-} \min }\right)$ : The minimal response time of LEDs and transistors can be calculated by $t_{r_{-} \text {min }}=\varnothing \times f_{c}^{-1}$, where $\emptyset$ is the duty cycle and $f_{c}$ is the flashing frequency at "cut-off”, obtained from a frequency response graph (i.e. Bode plot).

- Photosynthetic efficiency referrers to how much light (e.g. amount in photons or energy) is required by a phototroph to take up $\mathrm{CO}_{2}$ or produce $\mathrm{O}_{2}$ through photosynthesis (e.g. $\mu \mathrm{mol} \mathrm{CO}_{2}$ or $\mathrm{O}_{2}$ per $\mu \mathrm{mol}$ of photons). Effects of flashing light on the photosynthetic efficiency of single cells or chloroplasts and whole cultures should be distinct. Dilute cultures with narrow light paths and negligible light attenuation are usually used to identify effects of flashing light on single cells or chloroplasts (biological boundary). However, flashing light was mostly found to improve photosynthetic efficiency of whole microalgal cultures with high light attenuation (e.g. highly concentrated cultures).

- Photo- and mixotrophy: Phototrophic organisms use light as energy source to fix inorganic carbon dioxide in organic compounds. Heterotrophic organisms obtain energy 
and carbon from organic sources (glucose or acetate). A few mixotrophic microalgae are able to obtain energy and carbon skeletons by means of photosynthesis, active predation, endocytosis, and membrane-bound transport systems. Some others are even able to steal chloroplasts from other microalgae using a mechanism called myzocytosis.

- Pulse-width modulation is a tool used to control the power supply (e.g. dimming) of electrical devices such as LEDs. It generates a pulse wave signal (i.e., rectangular pulse wave) with an asymmetrical shape (i.e. the duration of the on-off cycle) described by the duty cycle. 
526 1. Communication from the comission to the european parliament, the council, the european 527 economic and social committee and the committee of the regions- Blue Growth: opportunities for 528 marine and maritime sustainable growth, European Comission, 2012.

529 2. Enzing, C. et al., Microalgae-based products for the food and feed sector: an outlook for Europe, J R

530 C Scientific and Policy reports, European Comission, 2014.

531 3. Ruiz, J. et al. (2016) Towards industrial products from microalgae. Energy \& Environmental Science.

532 4. Blanken, W. et al. (2013) Cultivation of microalgae on artificial light comes at a cost. Algal Res. 2, 533 333-340.

534 5. Schulze, P.S.C. et al. (2014) Light emitting diodes (LEDs) applied to microalgal production. Trends 535 Biotechnol. 32, 422-430.

536 6. Schulze, P.S.C. et al. (2016) Effect of light quality supplied by light emitting diodes (LEDs) on growth and biochemical profiles of Nannochloropsis oculata and Tetraselmis chuii. Algal Res. 16, 387-398.

7. Lee, C.-G. (1999) Calculation of light penetration depth in photobioreactors. Biotechnol. Bioproc. E. 4, 78-81.

8. MacKenzie, T.D. and Campbell, D.A. (2005) Cyanobacterial acclimation of rapidly fluctuating light is constrained by inorganic carbon status. J. Phycol. 41, 801-811.

9. Ooms, M.D. et al. (2016) Photon management for augmented photosynthesis. Nat. Commun. 7. 10. Park, K.-H. and Lee, C.-G. (2001) Effectiveness of flashing light for increasing photosynthetic efficiency of microalgal cultures over a critical cell density. Biotechnol. Bioproc. E. 6, 189-193.

11. Park, K.-H. and Lee, C.-G. (2000) Optimization of algal photobioreactors using flashing lights. Biotechnol. Bioproc. E. 5, 186-190.

12. Azevedo, I.L. et al. (2009) The transition to solid-state lighting. P. IEEE 97, 481-510.

13. Abu-Ghosh, S. et al. (2016) Flashing light in microalgae biotechnology. Bioresource Technol. 203, 357-363.

14. Iluz, D. et al. (2012) The enhancement of photosynthesis by fluctuating light. In Artifical Photosynthesis (Najafpour, M.M. ed), pp. 115-134.

15. Terry, K.L. (1986) Photosynthesis in modulated light: quantitative dependence of photosynthetic enhancement on flashing rate. Biotechnol. Bioeng. 28, 988-995.

16. Pearcy, R.W. (1990) Sunflecks and photosynthesis in plant canopies. Annu. Rev. Plant Biol. 41, 421453.

17. Jishi, T. et al. (2015) A kinetic model for estimating net photosynthetic rates of cos lettuce leaves under pulsed light. Photosynth. Res. 124, 107-116.

18. Hartmann, P. et al. (2014) The effect of photosynthesis time scales on microalgae productivity. Bioproc. Biosyst. Eng. 37, 17-25.

19. Vejrazka, C. et al. (2015) The role of an electron pool in algal photosynthesis during sub-second light-dark cycling. Algal Res. 12, 43-51.

20. Vejrazka, C. et al. (2012) Photosynthetic efficiency of Chlamydomonas reinhardtii in attenuated, flashing light. Biotechnol. Bioeng. 109, 2567-2574.

21. Vejrazka, C. et al. (2011) Photosynthetic efficiency of Chlamydomonas reinhardtii in flashing light. Biotechnol. Bioeng. 108, 2905-2913.

22. Vejrazka, C. et al. (2013) Photosynthetic efficiency and oxygen evolution of Chlamydomonas reinhardtii under continuous and flashing light. Appl. Microbiol. Biotechnol. 97, 1523-1532.

23. Brindley, C. et al. (2010) The oxygen evolution methodology affects photosynthetic rate measurements of microalgae in well-defined light regimes. Biotechnol. Bioeng. 106, 228-237.

24. Matthijs, H.C. et al. (1996) Application of light-emitting diodes in bioreactors: Flashing light effects and energy economy in algal culture (Chlorella pyrenoidosa). Biotechnol. Bioeng. 50, 98-107.

25. Nedbal, L. et al. (1996) Microscopic green algae and cyanobacteria in high-frequency intermittent light. J. Appl. Phycol. 8, 325-333.

26. Tennessen, D.J. et al. (1995) Efficiency of photosynthesis in continuous and pulsed light emitting diode irradiation. Photosynth. Res. 44, 261-269. 
27. Sager, J.C. and Giger, W. (1980) Re-evaluation of published data on the relative photosynthetic efficiency of intermittent and continuous light. Agr. Meteorol. 22, 289-302. 28. Varela, J.C. et al. (2015) Production of carotenoids by microalgae: achievements and challenges. Photosynth. Res. 125, 423-436.

580 29. Nishiyama, Y. and Murata, N. (2014) Revised scheme for the mechanism of photoinhibition and its 581 application to enhance the abiotic stress tolerance of the photosynthetic machinery. Appl. Microbiol. 582 Biotechnol. 98, 8777-8796.

583 30. Porcar-Castell, A. and Palmroth, S. (2012) Modelling photosynthesis in highly dynamic environments: the case of sunflecks. Tree Physiol. 32, 1062-1065.

31. Pearcy, R.W. and Way, D.A. (2012) Two decades of sunfleck research: looking back to move forward. Tree Physiol. 32, 1059-1061.

32. Nelson, N. and Junge, W. (2015) Structure and energy transfer in photosystems of oxygenic photosynthesis. Annu. Rev. Biochem. 84.

33. Goss, R. and Lepetit, B. (2015) Biodiversity of NPQ. J. Plant Physiol. 172, 13-32.

34. Hüner, N.P. et al. (2012) Chloroplast redox imbalance governs phenotypic plasticity: the "grand design of photosynthesis" revisited. Front. Plant Sci. 3.

35. Vinyard, D.J. et al. (2013) Photosystem II: the reaction center of oxygenic photosynthesis*. Annual review of biochemistry $82,577-606$.

36. Allahverdiyeva, Y. et al. (2013) Flavodiiron proteins Flv1 and Flv3 enable cyanobacterial growth and photosynthesis under fluctuating light. PNAS 110, 4111-4116.

37. Allahverdiyeva, Y. et al. (2014) Photoprotection of photosystems in fluctuating light intensities. J. Exp. Bot. 66, 2427-2436.

38. Dang, K.-V. et al. (2014) Combined increases in mitochondrial cooperation and oxygen photoreduction compensate for deficiency in cyclic electron flow in Chlamydomonas reinhardtii. Plant Cell 26, 3036-3050.

39. Rochaix, J.-D. (2011) Regulation of photosynthetic electron transport. BBA-Bioenergetics 1807, 878-886.

40. Krasnovsky, A. and Kovalev, Y.V. (2014) Spectral and kinetic parameters of phosphorescence of triplet chlorophyll a in the photosynthetic apparatus of plants. Biochemistry-US 79, 349.

41. Erickson, E. et al. (2015) Light stress and photoprotection in Chlamydomonas reinhardtii. Plant J. 82, 449-465.

42. Leakey, A. et al. (2002) Relative enhancement of photosynthesis and growth at elevated $\mathrm{CO}_{2}$ is greater under sunflecks than uniform irradiance in a tropical rain forest tree seedling. Plant, Cell Environ. 25, 1701-1714.

43. Papageorgiou, G.C. (2014) The non-photochemical quenching of the electronically excited state of chlorophyll a in plants: definitions, timelines, viewpoints, open questions. In Non-photochemical quenching and energy dissipation in plants, algae and cyanobacteria, pp. 1-44, Springer.

44. Takache, H. et al. (2015) Investigation of light/dark cycles effects on the photosynthetic growth of Chlamydomonas reinhardtii in conditions representative of photobioreactor cultivation. Algal Res. 8, 192-204.

45. Xue, S. et al. (2011) Growth of Spirulina platensis enhanced under intermittent illumination. J. Biotechnol. 151, 271-277.

46. Gris, B. et al. (2014) Cultivation of Scenedesmus obliquus in photobioreactors: effects of light intensities and light-dark cycles on growth, productivity, and biochemical composition. Appl. Biochem. Biotechnol. 172, 2377-2389.

47. Mouget, J.-L. et al. (1995) Long-term acclimatization of Scenedesmus bicellularis to high-frequency intermittent lighting $(100 \mathrm{~Hz})$. II. Photosynthetic pigments, carboxylating enzymes and biochemical composition. J. Plankton Res. 17, 875-890.

48. Sforza, E. et al. (2012) Adjusted light and dark cycles can optimize photosynthetic efficiency in algae growing in photobioreactors. PloS one 7, e38975.

49. Sastre, R.M.R. ( 2010) Kopplung physiologischer und verfahrenstechnischer Parameter beim Wachstum und bei der Produktbildung der Rotalge Porphyridium purpureum, KIT Scientific Publishing. 
50. Combe, C. et al. (2015) Long-term adaptive response to high-frequency light signals in the unicellular photosynthetic eukaryote Dunaliella salina. Biotechnol. Bioeng.

630 51. Abu-Ghosh, S. et al. (2015) Flashing light enhancement of photosynthesis and growth occurs when 631 photochemistry and photoprotection are balanced in Dunaliella salina. Eur. J. Phycol. 50, 469-480.

632 52. Yoshioka, M. et al. (2012) Effect of high frequency of intermittent light on the growth and fatty acid 633 profile of Isochrysis galbana. Aquaculture 338, 111-117.

634 53. Chen, C.-Y. et al. (2015) Enhancing the production of eicosapentaenoic acid (EPA) from 635 Nannochloropsis oceanica $\mathrm{CY} 2$ using innovative photobioreactors with optimal light source 636 arrangements. Bioresource Technol. 191, 407-413.

637 54. Kim, C.W. et al. (2014) Energy-efficient cultivation of Chlamydomonas reinhardtii for lipid accumulation under flashing illumination conditions. Biotechnol. Bioproc. E. 19, 150-158. 55. Katsuda, T. et al. (2006) Effect of flashing light from blue light emitting diodes on cell growth and astaxanthin production of Haematococcus pluvialis. J. Biosci. Bioeng. 102, 442-446.

56. Sastre, R.M.R. (2010) Kopplung physiologischer und verfahrenstechnischer Parameter beim Wachstum und bei der Produktbildung der Rotalge Porphyridium purpureum, KIT Scientific Publishing. 57. Emerson, R. and Arnold, W. (1932) A separation of the reactions in photosynthesis by means of intermittent light. J. Gen. Physiol. 15, 391-420.

58. Radmer, R. and Kok, B. (1977) Photosynthesis: limited yields, unlimited dreams. Bioscience 27, 599605.

59. Stitt, M. and ZHU, X.G. (2014) The large pools of metabolites involved in intercellular metabolite shuttles in $\mathrm{C} 4$ photosynthesis provide enormous flexibility and robustness in a fluctuating light environment. Plant Cell Environ. 37, 1985-1988.

60. Raven, J.A. and Ralph, P.J. (2014) Enhanced biofuel production using optimality, pathway modification and waste minimization. J. Appl. Phycol., 1-31.

61. Martín-Girela, I. et al. (2017) Flashing light effects on $\mathrm{CO}_{2}$ absorption by microalgae grown on a biofilm photobioreactor. Algal Res. 25, 421-430.

62. Belgio, E. et al. (2014) Economic photoprotection in photosystem II that retains a complete lightharvesting system with slow energy traps. Nat. Commun. 5, doi: 10.1038/ncomms5433.

63. Olvera-Gonzalez, E. et al. (2013) Intelligent lighting system for plant growth and development. Comput. Electron. Agr. 92, 48-53.

64. Grobbelaar, J.U. et al. (1996) Influence of high frequency light/dark fluctuations on photosynthetic characteristics of microalgae photoacclimated to different light intensities and implications for mass algal cultivation. J. Appl. Phycol. 8, 335-343.

65. Liao, Q. et al. (2014) A novel photobioreactor generating the light/dark cycle to improve microalgae cultivation. Bioresource Technol. 161, 186-191.

66. Ley, A.C. and Mauzerall, D.C. (1982) Absolute absorption cross-sections for photosystem II and the minimum quantum requirement for photosynthesis in Chlorella vulgaris. BBA-Bioenergetics 680, 95106.

67. Brindley, C. et al. (2011) Analysis of light regime in continuous light distributions in photobioreactors. Bioresource Technol. 102, 3138-3148.

68. Lunka, A. and Bayless, D. (2013) Effects of flashing light-emitting diodes on algal biomass productivity. J. Appl. Phycol. 25, 1679-1685.

69. Sun, Y. et al. (2016) Integrating planar waveguides doped with light scattering nanoparticles into a flat-plate photobioreactor to improve light distribution and microalgae growth. Bioresource Technol. 220, 215-224.

70. Heining, M. et al. (2015) Internal illumination of photobioreactors via wireless light emitters: a proof of concept. J. Appl. Phycol. 27, 59-66.

71. Sutor, A. et al. (2014) Method for optimizing the field coils of internally illuminated photobioreactors. IEEE T. Magn. 50, 1-4.

72. Glemser, M. et al. (2016) Application of light-emitting diodes (LEDs) in cultivation of phototrophic microalgae: current state and perspectives. Appl. Microbiol. Biotechnol. 100, 1077-1088.

73. Brindley, C. et al. (2016) Light regime optimization in photobioreactors using a dynamic photosynthesis model. Algal Res. 16, 399-408. 
681 74. Grobbelaar, J.U. (2006) Photosynthetic response and acclimation of microalgae to light 682 fluctuations. In Algal Cultures Analogues of Blooms and Applications (Subba Rao, D.V. ed), pp. 671-683, 683 Science Publishers.

684 75. Kane, R. et al. (2002) Revolution in lamps: A chronicle of 50 years of progress., Fairmount Press.

685 76. Grodzki, L. (2013) The Comparison of the Pulse and Constant-current LED Driving. Prz. 686 Elektrotechniczn. 89, 298-300.

687 77. Cape, J.L. et al. (2006) Understanding the cytochrome bc complexes by what they don't do. The Q688 cycle at 30. Trends Plant sci. 11, 46-55.

689 78. Römer, F. and Witzigmann, B. (2014) Effect of Auger recombination and leakage on the droop in 690 InGaN/GaN quantum well LEDs. Optics express 22, A1440-A1452.

691 79. Chow, C. et al. (2012) Digital signal processing for light emitting diode based visible light 692 communication. IEEE Photon. Soc. Newslett. 26, 9-13.

693 80. Park, J. (2010) Speedup of dynamic response of organic light-emitting diodes. J. Lightwave Technol. $69428,2873-2880$.

695 81. Rose, J. et al. (2010) Driving LED in a nanosecond regime by a fast operational amplifier. arXiv 696 preprint arXiv:1011.1954.

697 82. Janjua, B. et al. (2015) Going beyond 4 Gbps data rate by employing RGB laser diodes for visible 698 light communication. Opt. express 23, 18746-18753.

699 83. Chen, S.-F. et al. (2014) High-frequency modulation of GaAs/AIGaAs LEDs using Ga-doped ZnO current spreading layers. Electron Devic. Lett. 35, 36-38. 84. Wishkerman, A. and Wishkerman, E. (2017) Application note: A novel low-cost open-source LED system for microalgae cultivation. Comput. Electron. Agr. 132, 56-62. 85. Peltier, G. et al. (2010) Auxiliary electron transport pathways in chloroplasts of microalgae. Photosynth. Res. 106, 19-31. 